unhealthy behaviors and body mass index (BMI) in the relationship between high job strain and self-rated poor health in workers with a low educational level.

Methods A total of 8,369 low educated workers, who participated in the Lifelines cohort study during the period 20122017, were included. Self-reported job strain, health behaviors (smoking, physical activity, fruit and vegetable consumption) and BMI were assessed at baseline, and self-rated health after 2 years. To assess mediation by the health behaviors and BMI, structural equation modeling with logistic and multinomial regression analyses were performed.

Results Workers with high job strain had a higher odds of poor health (OR 1.34; 95\%CI 1.13-1.60) compared to those with low job strain. Workers with high job strain were more likely to have a lack of physical activity (OR 1.14; 95\%CI 1.01-1.28), but were not more likely to smoke, to be overweight or obese, or to have a low fruit or vegetable consumption. Workers who smoked (OR 1.37; 95\%CI 1.161.60 ), had a lack of physical activity (OR 1.25; 95\%CI $1.08-1.43$ ) or were overweight (OR 1.37; 95\%CI 1.16-1.61) or obese (OR 2.25; 95\%CI 1.86-2.72) were more likely to report poor health. Indirect (mediating) effects of unhealthy behaviors and BMI in the relationship between high job strain and poor health were small and not statistically significant.

Conclusion No mediating effects of unhealthy behaviors or BMI were found in the relationship between high job strain and self-rated poor health among workers with a low educational level.

\section{0-43 CHANGES IN JOB CONTROL AND PERCEPTIONS OF GENERAL HEALTH: A LONGITUDINAL ANALYSIS OF AUSTRALIAN WORKERS, 2005-2017}

${ }^{1}$ Yamna Taouk, Matthew Spittal, George Disney, Anthony LaMontagne. ${ }^{1}$ University of Melbourne, Australia

\subsection{6/OEM-2021-EPI.71}

Introduction Low job control is associated with poor health outcomes. However, establishing the causal nature of this association is challenging mostly due to methodological biases across studies.

Objectives To examine whether changes in job control over time were associated with changes in perceptions of general health, using data from a nationally representative longitudinal study of Australian households.

Methods Our sample included 105,017 observations (18,574 persons) over 13 years from working age participants with information on job control, general health, and other sociodemographic and health factors. We used dynamic fixed-effects regression models to control for time-invariant confounding and reverse causation, and ordinary least squares regression with cumulative job control exposure to analyse cumulative exposure to low job control. Perceptions of general health, scores ranging from 0-100 points (higher scores indicating better health), were modelled in relation to self-reported job control.

Results There was a strong stepwise relationship between increasing job control and general health, with a 2.4-point increase in general health $(95 \%$ CI 2.0-2.8) for participants in the highest job control quintile compared to those in the lowest quintile. The inclusion of a lagged effect of job control supported a small prospective relationship $(\beta=0.17 ; 95 \%$ CI $0.07-0.27)$ but the contemporaneous association was essentially unchanged. Going from the lowest to highest observed value of cumulative job control predicted an 8-point higher general health score supporting a prospective association between job control and general health over a period of 4 years $(\beta=0.47$; 95\% CI $0.38-0.57)$.

Conclusion This analysis with improved causal inference over previous research showed that increased job control is strongly associated with increasing general health. The validation of job control as a potentially modifiable causal factor of general health has considerable public health implications, and substantial economic consequences. Appropriate work stress interventions might contribute to better health and well-being.

\section{0-265 LATENT PROFILE ANALYSIS: AN ALTERNATIVE PERSPECTIVE ON JOB SUB-TYPES OF THE JOB DEMAND-CONTROL-SUPPORT MODEL}

${ }^{1}$ Igor Portoghese, Marcello Campagna. 'University of Cagliari, Italy

\subsection{6/OEM-2021-EPI.72}

Introduction The Job Demand-Control-Support (JDCS) model suggests that a combination of job demands (JD), job control (JC), and job support (JS) forms eight different job types. In the last three decades, these constellations of job types have been explored by adopting the traditional variable-centered perspective. In this sense, these constellations of job types as postulated in the JDCS model have been rarely confirmed empirically and few studies were able to find all the hypothesized constellations.

Objectives The aim of this study was to expand previous research on the JDCS model by adopting a person-centered approach to identify relevant job type profiles.

Methods We applied latent profile analysis (LPA) to identify profiles in a sample of Italian workers $(n=348)$. LPA including one to eight latent profiles were estimated by using MPlus 7 robust maximum likelihood estimator, considering the following goodness-of-fit indices: Bayesian information criterion, Akaike information criterion, Constant AIC; bootstrapped likelihood ratio p-value and the Lo-Mendell Rubin adjusted likelihood ratio.

Results LPA showed a five-latent-profiles solution: isolated high strain (high JD, low JC, low support; $\mathrm{n}=36,10.35 \%$ ), cowboy-hero (high JC, low support; $n=16,4.60 \%$ ), moderatestrain (moderate JD, moderate JC, low support; $\mathrm{n}=125$, 35.92\%), collective-low-strain (low JD, high JC, high support; $\mathrm{n}=37,10.63 \%$ ), and supported (high support, $\mathrm{n}=134$, $38.51 \%)$.

Conclusion Results from our study partially agree with JDCS theorization as it replicated three of the eight-profile solutions identified, finding two new constellations. In fact, we were not able to identify the passive jobs, active jobs, obedient comrade, and participatory leader constellations. This study extends previous research on JDCS, highlighting that future studies should consider empirically identified profiles rather than theoretically defined subgroups. Finally, one of the main theoretical contribution of the present study was the consideration of job support as an important component for job types identification, as postulated in the JDCS model. 